BMJ Open

Diabetes

Research

\& Care

\title{
Pen needle design influences ease of insertion, pain, and skin trauma in subjects with type 2 diabetes
}

\author{
Kezia A Præstmark, ${ }^{1,2}$ Morten L Jensen, ${ }^{3}$ Nils B Madsen, ${ }^{2}$ Jonas Kildegaard, ${ }^{4}$ \\ Bente M Stallknecht ${ }^{1}$
}

To cite: Præstmark KA, Jensen ML, Madsen NB, et al. Pen needle design influences ease of insertion, pain, and skin trauma in subjects with type 2 diabetes. BMJ Open Diabetes Research and Care 2016;4: e000266. doi:10.1136/ bmjdrc-2016-000266

Received 10 May 2016 Revised 12 October 2016 Accepted 29 October 2016

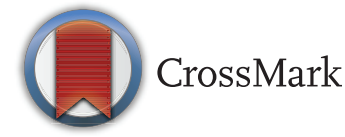

${ }^{1}$ Department of Biomedical Sciences, University of Copenhagen, Copenhagen, Denmark

2Device R\&D Device R\&D, Novo Nordisk A/S, Hillerød, Denmark

${ }^{3}$ Medical \& Science Devices, Novo Nordisk A/S, Søborg, Denmark

${ }^{4}$ Insulin Pharmacology, Novo Nordisk A/S, Måløv, Denmark

Correspondence to Dr Kezia A Præstmark; kezj@novonordisk.com

\section{ABSTRACT}

Objective: Pen needles used for subcutaneous injections have gradually become shorter, thinner and more thin walled, and thereby less robust to patient reuse. Thus, different needle sizes, alternative tip designs and needles resembling reuse were tested to explore how needle design influences ease of insertion, pain and skin trauma.

Research design and methods: 30 subjects with injection-treated type 2 diabetes and body mass index $25-35 \mathrm{~kg} / \mathrm{m}^{2}$ were included in the single-blinded study. Each subject received abdominal insertions with 18 different types of needles. All needles were tested twice per subject and in random order. Penetration force (PF) through the skin, pain perception on $100 \mathrm{~mm}$ visual analog scale, and change in skin blood perfusion (SBP) were quantified after the insertions.

Results: Needle diameter was positively related to PF and SBP $(p<0.05)$ and with a positive pain trend relation. Lack of needle lubrication and small 'needle hooks' increased PF and SBP $(p<0.05)$ but did not affect pain. Short-tip, obtuse needle grinds affected PF and SBP, but pain was only significantly affected in extreme cases. PF in skin and in polyurethane rubber were linearly related, and pain outcome was dependent of SBP increase.

Conclusions: The shape and design of a needle and the needle tip affect ease of insertion, pain and skin trauma. Relations are seen across different data acquisition methods and across species, enabling needle performance testing outside of clinical trials. Trial registration number: NCT02531776; results.

\section{INTRODUCTION}

There are $\sim 387$ million people with diabetes mellitus in the world ${ }^{1}$ and it has been estimated that more than 100 million insulin injections are performed worldwide every day. ${ }^{2-4}$ Thus, it is important that needles for subcutaneous administration of antidiabetic drugs are developed to be as safe, pain-free, and comfortable to use as possible.

Needle manufacturers have through the years sought to develop needles which cause less pain and less skin trauma, mainly by decreasing needle length and diameter; however, it is a challenge to maintain a

\section{Significance of this study}

What is already known about this subject?

- Shorter and thinner needles cause less pain, thinner needles cause less penetration force, and a five-grind needle tip has previously shown to cause less penetration force.

\section{What are the new findings?}

- Needle diameter and obtuse tip designs were positively related to skin penetration force, pain, and skin trauma.

- Minor side effects of reuse (worn-off silicone and small hooks) may not necessarily affect user experience.

How might these results change the focus of research or clinical practice?

- Smaller needle diameter and obtuse tip designs should be used in combination to design more robust needles for people with diabetes.

- Since skin trauma and pain perception after needle insertions relate positively to each other, skin trauma in animal models can be used to evaluate performance when developing new needles.

sufficiently large inner lumen to keep an acceptable low injection force. This can cause the wall thickness to be critically small, which challenges the robustness of the needles, and increases the risk of needle tip damage during, for example, cap removal or handling. Thus, it is of interest to investigate how more robust alternatives to the traditional three-grind needle (ie, a needle tip with three 'edges' or grind angles) impact the user experience. Even though a few needles with alternate grinds are marketed, little is known about how needle tip design influences ease of insertion through skin, pain, and tissue trauma.

Needle performance is usually measured by pain perception of test subjects in a clinical trial ${ }^{5-10}$ or by mechanical tests with measurement of penetration force (PF) through polyurethane rubber (PUR). ${ }^{5} \quad 11-13$ 
However, pain is a subjective measure with many individual frames of reference causing high data variance, and clinical trials are costly and time-consuming. Furthermore, it is unknown if and how PF through PUR relates to ease of insertion through skin, pain, or other biological factors.

This study quantitates PF, perceived pain, and the small skin trauma which occurs at a needle penetration. Skin trauma was previously explored on pigs by the authors $^{14}$ by using Laser Speckle Contrast Analysis (LASCA) technology ${ }^{15-18}$ to measure the increase of skin blood perfusion (SBP) following pen needle insertions, but it is unknown if and how SBP relates to pain and ease of skin penetration in humans.

The aims of this study were to explore how various designs of $32 \mathrm{G}$ and $34 \mathrm{G}$ needles influence $\mathrm{PF}$, pain, and SBP, explore the relation between pain and SBP, and explore the relation between PF in PUR and skin.

\section{RESEARCH DESIGN AND METHODS \\ Study design}

The study was a single-center, one-visit, single-blinded trial in people with type 2 diabetes, investigating skin PF, pain, and SBP during needle insertion into the subcutis on the abdomen with different needle designs.

\section{Subjects}

The trial sample consisted of 30 Caucasian men and women aged 18-70 years with type 2 diabetes, body mass index (BMI) $25-35 \mathrm{~kg} / \mathrm{m}^{2}$, and self-injecting antiglycemic drugs (insulin formulation or glucagon-like peptide 1 agonist) for more than 6 months. Subjects were excluded if they administered substances which could influence pain perception. The subjects' demographics were recorded, and information about their daily treatment and regular use of needles was collected.

\section{Randomization}

All eligible subjects would receive the same number and type of needle insertions. Each subject drew a number on arrival which determined the prerandomized needle and insertion site sequence.

\section{Needle insertions}

With the subject in the supine position, 36 fields were drawn-18 on each side of the umbilicus. A screen was placed above the chest to blind the subject for the needles. Three test insertions were performed outside of the fields in order to prepare the subject and introduce the evaluation procedure of pain perception. Eighteen different needles were included in the trial, and all needles were tested twice on each subject, once on each side of the abdomen. No substance was injected.

\section{Needles used in the study}

Eighteen needles with different designs or properties were included in the study; see table 1 for details and illustrations of the needles. Five of the needles were marketed needles; NovoFine 32G tip $6 \mathrm{~mm}$ (NF-32), NovoFine 30G $6 \mathrm{~mm}$ (NF-30), NovoFine 28G $12.7 \mathrm{~mm}$ (NF-28; all Novo Nordisk A/S, Bagsværd, Denmark), BD Micro-Fine Ultra Pentapoint 32G 4 mm (BD-32; Becton, Dickinson and Company, Franklin Lakes, New Jersey, USA), and NANOPASS 34G $4 \mathrm{~mm}$ (TER-34; Terumo Corporation, Tokyo, Japan). The BD-32 is a five-grind needle (ie, five edges/grind angles on the needle tip) ${ }^{5}$ and the TER-34 needle has an asymmetrical three-grind tip. ${ }^{19}$ Furthermore, the NF-32 and the TER-34 needles are both tapered needles with NF-32 being $31 \mathrm{G}$ at the needle base $(0.015 \mathrm{~mm}$ increase) and TER-34 being $32 \mathrm{G}$ at the needle base $(0.06 \mathrm{~mm}$ increase $)$. The NF-32 needle has a traditional three-grind needle tip.

Ten needles were custom-designed needles manufactured, lubricated, sterilized and packed by Hart Needles (Sparta, Michigan, USA). Two were needles with the same standard three-grind as NF-32, but non-tapered; 32G (HN-32) and 34G (HN-34). Two were three-grind short-tip needles; 32G (HN3-32) and 34G (HN3-34). Two were two-grind (ie, two edges/grind angles on the needle tip) short-tip needles; 32G (HN2-32) and 34G (HN2-34). Two were one-grind (ie, one edge/grind angle on the needle tip) short-tip needles; 32G (HN1SH-32) and 34G (HN1SH-34). Two were one-grind long-tip needles; 32G (HN1LO-32) and 34G (HN1LO-34).

Although pen needles are for single use only, the majority of users reuse the needles, ${ }^{20}{ }^{21}$ so the silicone is at risk of being worn-off, and the needle tip might get hooked from handling, recapping, etc. Therefore, three of the needles were modified NF-32 needles which were either tip damaged with a hook formation or had the silicone removed. The modifications were made in a laminar flow bench, examined under a microscope in a clean laboratory, and individually repacked and autoclaved before use in the trial. One had the silicone coating removed (NOSILIC) by repeated wiping with 82\% ethanol swabs (Mediq Danmark A/S, Brøndby, Denmark). Two needles had hooks, 50 and $150 \mu \mathrm{m}$, respectively. The needle with a $50 \mu \mathrm{m}$ hook (HOOK50) was included because a pilot laboratory test of typical handling errors potentially damaging the needle tip, including drops, oblique cap removals, and attachment revealed that the typical hook formation is in the range $40-60 \mu \mathrm{m}$. The needle with a large hook of $150 \mu \mathrm{m}$ (HOOK150) was included to compare human data with previous SBP measurements in pigs. ${ }^{14}$

\section{Assessments}

\section{Penetration force}

Skin PF was measured using a handheld force gauge instrument (Advanced Force Gauge 5N, Mecmesin, Slinfold, UK), connected to a laptop recording the PF during the needle insertion through the skin. The peak value of the PF was used for further data analysis. PUR PF was measured using a Lloyd-Ametek LR5KPLUS Material Testing Machine (Lloyd Instruments, West 
Table 1 Overview of the 18 different needles used in the study

\begin{tabular}{|c|c|c|c|c|c|}
\hline Needle & Brand name & Gauge & $\begin{array}{l}\text { Number } \\
\text { of grinds }\end{array}$ & Modification & Design illustration \\
\hline \multicolumn{6}{|c|}{ Marketed needles } \\
\hline NF-32 & NovoFine & $32 \mathrm{G}$ tip (31G & 3 & - & \\
\hline \multirow[t]{2}{*}{ NF-30 } & & base) & & & \\
\hline & NovoFine & $30 G$ & 3 & - & \\
\hline NF-28 & NovoFine & $28 \mathrm{G}$ & 3 & - & \\
\hline BD-32 & $\begin{array}{l}\text { BD Micro-Fine Ultra } \\
\text { Pentapoint }\end{array}$ & $32 \mathrm{G}$ & 5 & - & Please see (5) \\
\hline TER-34 & Nanopass & $\begin{array}{l}34 \mathrm{G}(32 \mathrm{G} \\
\text { base) }\end{array}$ & & - & Please see (25) \\
\hline \multicolumn{6}{|c|}{ Custom-designed needles } \\
\hline HN-32 & - & $32 \mathrm{G}$ & 3 & - & Same tip design as \\
\hline $\mathrm{HN}-34$ & - & $34 \mathrm{G}$ & 3 & - & NF needles \\
\hline HN3-32 & - & $32 \mathrm{G}$ & 3 & - & \\
\hline HN3-34 & - & $34 G$ & 3 & - & \\
\hline HN2-32 & - & $32 \mathrm{G}$ & 2 & - & \\
\hline HN2-34 & - & $34 \mathrm{G}$ & 2 & - & \\
\hline HN1LO-32 & - & $32 G$ & 1 & - & \\
\hline HN1LO-34 & - & $34 G$ & 1 & - & \\
\hline HN1SH-32 & - & $32 \mathrm{G}$ & 1 & - & \\
\hline HN1SH-34 & - & $34 \mathrm{G}$ & 1 & - & \\
\hline \multicolumn{6}{|c|}{ Modified needles } \\
\hline NOSILIC & - & $32 \mathrm{G}$ tip & 3 & $\begin{array}{l}\text { Worn-off } \\
\text { silicone }\end{array}$ & $\begin{array}{l}\text { Same tip design as } \\
\text { NF-32 needles }\end{array}$ \\
\hline HOOK50 & - & $32 G$ tip & 3 & $50 \mu \mathrm{m}$ hook & $\longrightarrow$ \\
\hline HOOK150 & - & $32 G$ tip & 3 & $150 \mu \mathrm{m}$ hook & \\
\hline
\end{tabular}

Sussex, UK). Five of each needle types were included and peak PFs were used for comparison to peak PFs in skin. The test procedure is defined in the Chinese standard BG/T 15811-2001 and previously described. ${ }^{13}$

\section{Pain}

Immediately after each needle insertion the subjects rated pain intensity on a $0-100 \mathrm{~mm}$ visual analog scale (VAS), where the score 0 indicated no pain and the score 100 indicated the worst imaginable pain.

\section{Skin blood perfusion}

SBP was measured using a LASCA Pericam PSI (Perimed, Stockholm, Sweden), which was placed above the skin. Before needle insertions, a baseline of $3 \mathrm{~min}$ was recorded. Three needle insertions were performed for each recording. Immediately after removal of the third needle insertion and corresponding pain rating, the recording was resumed and continued for $15 \mathrm{~min}$. The 15 min signal recording was chosen based on previous experience with SBP measurements in pigs, ${ }^{14}$ and the need for test subjects to remain immobile during the LASCA recordings in order to minimize signal noise. The experiments were carried out to meet the LASCA experimental recommendations outlined by Mahé $e t a l^{22}$ to minimize procedure responsible variation in the recordings. A dedicated, thermally controlled room $\left(23 \pm 1^{\circ} \mathrm{C}\right)$ with constant lighting and low ventilation was used. The data sampling frequency was 10 images per second, and averaged over 10 images to an effective frame rate of one image per second. The LASCA data analysis was performed as described in previous tests on pigs. ${ }^{14}$ In brief, the mean value of each baseline recording was used as baseline reference. The following recorded SBP signal point for the $15 \mathrm{~min}$ recording was compared with this reference, and the SBP graph for each needle is expressed as a percentage of the baseline. Area under the curve (AUC) values of the SBP graphs were used for comparison between the different needles. 


\section{Statistical analysis}

Statistical analysis of data was performed using SAS JMP V.10.0.2 (SAS Institute, Cary, North Carolina, USA). Comparison of needles with respect to PF and SBP were expressed as ratios, and therefore data were log-transformed prior to statistical analysis, and differences on log scale were back-transformed to the original scale as ratios. Data were analyzed by an ANOVA model with subject, needle, region (left/right) and insertion field (A-H) as fixed effects, injection order as a covariate (not included in the model for $\mathrm{SBP}$ ), and needle $\times$ subject as a random effect. To evaluate effect relations, a model was fit with pain as response and subject, needle type, SBP and PF as model effects. Subject was set as random effect. Linear regression analysis was made to compare PF in skin and PUR.

\section{Ethics and authorizations}

The study received ethical approval by the Regional Committee of Danish Health Research Ethics (H-6-2014-042) and health authority approval by the Danish Health and Medicines Authority (journal number 2014071250, EUDAMED CIV-ID no. CIV-14-07-012361). All study participants signed informed consent forms before entering the study and were free to withdraw from the study at any time. The study was conducted in accordance with the Declaration of Helsinki of 1964, as revised in 2013, and the guidelines for Good Clinical Practice. ClinicalTrials.gov number NCT02531776.

\section{RESULTS}

Thirty people with type 2 diabetes participated in the study where $19(63 \%)$ were men, age of $59 \pm 6$ years (mean, SD), BMI of $31 \pm 3 \mathrm{~kg} / \mathrm{m}^{2}$ (mean, SD), diabetes duration of $13 \pm 7$ years (mean, SD), injection treatment duration of $7 \pm 5$ years (mean, SD), and $26(87 \%)$ of the test subjects performed all or some of their injections in the abdomen.

Out of the 1080 needle insertions, 75 were excluded from the final data set. The primary reason was that in some instances the large hook needle (HOOK150) and two-grind needles (HN2) did not penetrate the skin, which accounted for 65 of the exclusions. In addition, six were excluded because the needle was damaged before or during insertion, and four were excluded because of insertion procedure deviations.

Table 2 summarizes the values found for PF, SBP and pain for all needles in the study, and table 3 represents the comparisons between the needles.

\section{Needle design influence on effect parameters Needle diameter}

PF and SBP increased with needle diameter (table 3, 'Diameter'). Pain intensity seemed to increase with needle diameter, but the difference was not significant. Figure 1 shows the averaged SBP profiles in human skin for the 32G (NF-32), 30G (NF-30), 28G (NF-28), and large hook (HOOK150) needles, creating peak increases of $16 \%, 18 \%, 25 \%$, and $34 \%$, respectively. All profiles reach their peak between 1 and 4 min after the needle insertions. For all the custom-designed needles, the 34G versions also caused smaller peak $\mathrm{PF}$, increase in SBP, and pain intensities than their $32 \mathrm{G}$ equivalents (table 3 , 'Tip design').

Table 2 Values of different tests for all needles

\begin{tabular}{|c|c|c|c|}
\hline Needle & $\begin{array}{l}\text { PF (N) } \\
\text { LSM }(95 \% \mathrm{CI})\end{array}$ & $\begin{array}{l}\text { SBP (AUC PU) } \\
\text { LSM }(95 \% \text { CI) }\end{array}$ & $\begin{array}{l}\text { Pain (mm on } 100 \mathrm{~mm} \text { VAS) } \\
\text { LSM }(95 \% \mathrm{Cl})\end{array}$ \\
\hline NF-28 & $0.32(0.30$ to 0.34$)$ & 235 (201 to 275$)$ & $19.2(14.2$ to 24.1$)$ \\
\hline NF-30 & $0.29(0.27$ to 0.30$)$ & 161 (138 to 190$)$ & 15.0 (10.1 to 20.0$)$ \\
\hline NF-32 & $0.25(0.23$ to 0.26$)$ & 155 (133 to 182$)$ & 14.6 (9.6 to 19.6$)$ \\
\hline BD-32 & $0.16(0.15$ to 0.17$)$ & 182 (156 to 213$)$ & $13.0(8.1$ to 18.0$)$ \\
\hline TER-34 & $0.17(0.16$ to 0.19$)$ & 152 (130 to 178$)$ & 13.3 (8.4 to 18.3$)$ \\
\hline $\mathrm{HN}-32$ & $0.48(0.45$ to 0.51$)$ & 211 (181 to 247$)$ & 15.9 (10.9 to 21.0$)$ \\
\hline $\mathrm{HN}-34$ & $0.40(0.37$ to 0.42$)$ & 165 (140 to 194$)$ & $18.3(13.3$ to 23.4$)$ \\
\hline HN3-32 & 1.05 (0.99 to 1.12$)$ & 339 (288 to 398$)$ & 22.7 (17.5 to 27.8$)$ \\
\hline HN3-34 & $0.65(0.61$ to 0,69$)$ & 270 (229 to 318$)$ & 20.1 (15.0 to 25.2 ) \\
\hline HN2-32 & $1.44(1.34$ to 1.55$)$ & 370 (306 to 447$)$ & 27.9 (22.0 to 33.8$)$ \\
\hline HN2-34 & 0.87 (0.81 to 0.93$)$ & 298 (250 to 356$)$ & 22.5 (16.8 to 28.3 ) \\
\hline HN1LO-32 & 0.65 (0.61 to 0.69$)$ & 300 (256 to 351$)$ & 18.1 (13.2 to 23.1$)$ \\
\hline HN1LO-34 & $0.64(0.60$ to 0.68$)$ & 244 (207 to 289) & $14.2(9.1$ to 19.3$)$ \\
\hline HN1SH-32 & $1.03(0.91$ to 1.10$)$ & 369 (314 to 433$)$ & 22.7 (17.5 to 27.9$)$ \\
\hline HN1SH-34 & $0.77(0.72$ to 0.82$)$ & 267 (227 to 313$)$ & 21.2 (16.2 to 26.3$)$ \\
\hline HOOK50 & $0.48(0.45$ to 0.51$)$ & 215 (184 to 252$)$ & 18.1 (15.2 to 23.1$)$ \\
\hline HOOK150 & 1.36 (1.27 to 1.46$)$ & 386 (321 to 463$)$ & 27.1 (21.1 to 33.0 ) \\
\hline NOSILIC & $0.37(0.35$ to 0.39$)$ & 203 (173 to 237$)$ & $14.5(9.5$ to 19.4$)$ \\
\hline
\end{tabular}

PF data measured in Newton, SBP data measured as AUC of recorded PUs, and pain data measured on a $100 \mathrm{~mm}$ VAS. All values are given as the LSM with $95 \% \mathrm{Cl}$.

AUC, area under the curve; LSM, least square mean; PF, penetration force; PU, perfusion unit; SBP, skin blood perfusion; VAS, visual analog scale. 
Tip design

The standard three-grind 32G needle (NF-32) caused a larger peak $\mathrm{PF}$ than the asymmetrical three-grind tapered 34G (TER-34) and the five-grind 32G (BD-32) needles (table 3, 'Tip design, Asymmetrical 3-grind and 3 -grind vs 5-grind'). No difference was seen in peak $\mathrm{PF}$ between the BD-32 and the TER-34 needle. The needles did not differ in SBP or in pain intensity.

In general, all the custom-designed needles caused larger peak $\mathrm{PF}(\mathrm{p}<0.05)$ than the $32 \mathrm{G}$ needle with standard three-grind (HN-32; table 3, 'Tip design'). Apart from the one-grind long-tip 34G needle (HN1LO-34), all the custom-designed needles also caused a larger increase in SBP $(p<0.05)$ than the HN-32 needle. Pain intensity was only statistically larger for the two-grind 32G needle (HN2-32) compared with the HN-32 needle (table 3, 'Tip design, Standard 3-grind vs 2-grind'). The HN1LO-34 needle was the only needle causing less pain than the HN-32 needle.

\section{Worn-off silicone}

Insertion with a needle with worn-off silicone (NOSILIC) caused higher PF and increase in SBP than a new fully lubricated needle (table 3, 'Worn-off silicone'). Worn-off silicone did not influence pain intensity.
Hooks

The small hook of $50 \mu \mathrm{m}$ (HOOK50) caused higher PF and SBP than a needle without hook (table 3, 'Hooks'), but did not cause higher pain intensity, whereas the large hook (HOOK150) caused higher PF, larger SBP increase, and higher pain intensity.

\section{Effect relations}

We wished to explore whether needle pain can be predicted by PF and/or SBP. A least square mean scatter plot matrix is seen in figure 2, where a linear approximation is fitted to show the relations between PF, pain intensity, and SBP. It is seen that all effects are positively related. The fitted model estimate that $59 \%$ of the influence on pain comes from intersubject variability. Next after the subject effect, the SBP is the effect most significantly predicting pain $(\mathrm{p}=0.0002)$, and then $\mathrm{PF}$ $(\mathrm{p}=0.0154)$.

\section{PF in skin versus PUR}

PFs of all 18 needles measured higher in PUR than in skin, and PF in PUR and skin were positively related (PF in $\mathrm{PUR}=1.3847 \times \mathrm{PF}$ in skin $+0.1173, \mathrm{R}^{2}=0.86, \mathrm{p}<0.0001$ ).

Table 3 Needle comparisons for different tests

\begin{tabular}{|c|c|c|c|c|c|c|c|c|}
\hline \multirow[b]{3}{*}{ Test } & \multirow[b]{3}{*}{ Needle 1} & \multirow[b]{3}{*}{ Needle 2} & \multicolumn{6}{|c|}{ Estimated difference or ratio } \\
\hline & & & \multicolumn{2}{|l|}{$\overline{\mathbf{P F}}$} & \multicolumn{2}{|l|}{ SBP } & \multicolumn{2}{|l|}{ Pain } \\
\hline & & & Ratio & p Value & Ratio & p Value & $\begin{array}{l}\text { Ditrerence } \\
\text { (mm) }\end{array}$ & p Value \\
\hline \multirow[t]{3}{*}{ Diameter } & NF-32 & NF30 & $1.16^{\star}$ & $<0.01$ & 1.04 & 0.72 & 0.44 & 0.90 \\
\hline & NF-32 & NF28 & $1.29^{\star}$ & $<0.01$ & $1.51^{\star}$ & $<0.01$ & 4.58 & 0.20 \\
\hline & NF-30 & NF28 & $1.11^{\star}$ & $<0.05$ & $1.45^{\star}$ & $<0.01$ & 4.13 & 0.25 \\
\hline \multicolumn{9}{|l|}{ Tip design } \\
\hline \multirow[t]{2}{*}{ Asymmetrical 3-grind } & NF-32 & TER-34 & $0.71^{*}$ & $<0.01$ & 0.97 & 0.85 & -1.28 & 0.72 \\
\hline & BD-32 & TER-34 & 1.08 & 0.06 & 0.83 & 0.11 & 0.28 & 0.94 \\
\hline 3-grind vs 5-grind & NF-32 & BD-32 & $0.66^{*}$ & $<0.01$ & 1.17 & 0.16 & -1.56 & 0.66 \\
\hline \multirow{3}{*}{ Standard 3-grind vs short 3-grind } & $\mathrm{HN}-32$ & HN3-32 & $2.18^{*}$ & $<0.01$ & $1.60^{\star}$ & $<0.01$ & 6.71 & 0.067 \\
\hline & HN-32 & HN3-34 & $1.34^{*}$ & $<0.01$ & $1.28^{*}$ & 0.03 & 4.14 & 0.26 \\
\hline & HN3-32 & HN3-34 & $0.62^{*}$ & $<0.01$ & 0.80 & 0.05 & -2.58 & 0.48 \\
\hline \multirow[t]{3}{*}{ Standard 3-grind vs short 2-grind } & $\mathrm{HN}-32$ & HN2-32 & $3.00^{*}$ & $<0.01$ & $1.75^{\star}$ & $<0.01$ & $11.97^{*}$ & $<0.01$ \\
\hline & $\mathrm{HN}-32$ & HN2-34 & $1.80^{*}$ & $<0.01$ & $1.41^{*}$ & $<0.01$ & 6.58 & 0.09 \\
\hline & HN2-32 & HN2-34 & $0.60^{*}$ & $<0.01$ & 0.81 & 0.10 & -5.39 & 0.20 \\
\hline \multirow[t]{6}{*}{ Standard 3-grind vs short 1 -grind } & HN-32 & HN1LO-32 & $1.34^{\star}$ & $<0.01$ & $1.42^{\star}$ & $<0.01$ & 2.17 & 0.55 \\
\hline & HN-32 & HN1LO-34 & $1.33^{\star}$ & $<0.01$ & 1.15 & 0.22 & -1.72 & 0.64 \\
\hline & HN1LO-32 & HN1LO-34 & 0.99 & 0.89 & 0.81 & 0.082 & -3.89 & 0.29 \\
\hline & HN-32 & HN1SH-32 & $2.14^{\star}$ & $<0.01$ & $1.74^{\star}$ & $<0.01$ & 6.77 & 0.07 \\
\hline & HN-32 & HN1SH-34 & $1.59^{\star}$ & $<0.01$ & $1.26^{\star}$ & 0.04 & 5.28 & 0.15 \\
\hline & HN1SH-32 & HN1SH-34 & $0.74^{*}$ & $<0.01$ & $0.72^{\star}$ & $<0.01$ & -1.49 & 0.69 \\
\hline Worn-off silicone & NF-32 & NOSILIC & $1.51^{*}$ & $<0.01$ & $1.30^{\star}$ & $<0.05$ & 0.137 & 0.96 \\
\hline \multirow[t]{3}{*}{ Hooks } & NF-32G & HOOK50 & $1.94^{\star}$ & $<0.01$ & $1.38^{\star}$ & $<0.01$ & 3.54 & 0.33 \\
\hline & NF-32G & HOOK150 & $5.55^{\star}$ & $<0.05$ & $2.48^{\star}$ & $<0.01$ & $12.45^{\star}$ & $<0.01$ \\
\hline & HOOK50 & HOOK150 & $2.86^{\star}$ & $<0.01$ & $1.79^{\star}$ & $<0.01$ & $8.91^{*}$ & $<0.05$ \\
\hline
\end{tabular}

Between-needle comparisons of peak PF and increase of SBP, respectively, are expressed as a ratio. A ratio value higher than 1.00 indicates that needle 2 caused higher PF or SB than needle 1. Pain comparisons are expressed as a difference in mm on a $100 \mathrm{~mm}$ VAS scale. A positive value indicates that needle 2 rated higher on pain than needle 1. ${ }^{*}$ Comparison is statistically different $(p<0.05)$.

PF, penetration force; SBP, skin blood perfusion; VAS, visual analog scale. 
Figure 1 SBP following insertions of needles with different diameters. Averaged (mean \pm SEM) SBP profiles measured 0-15 min after needle insertion. Only $1 \mathrm{~min}$ of the $3 \mathrm{~min}$ baseline is included in the plot. SBP, skin blood perfusion.

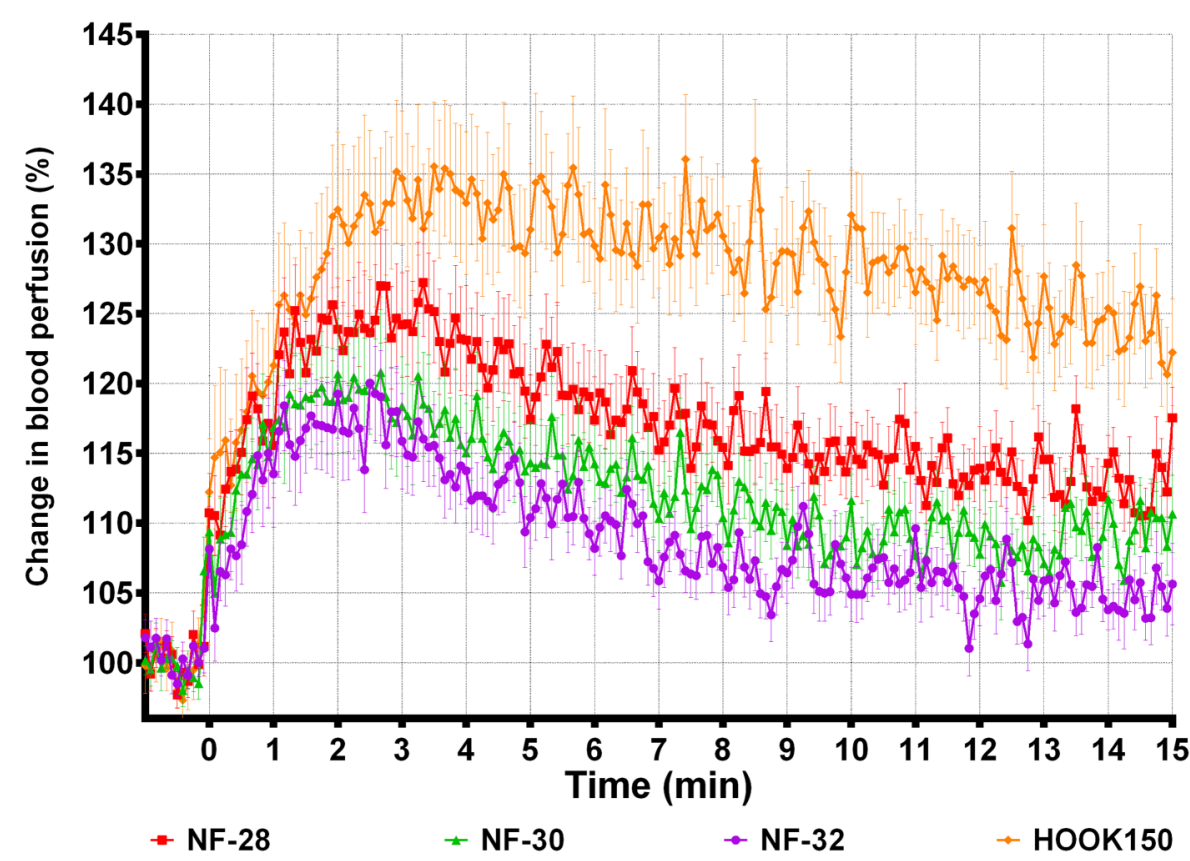

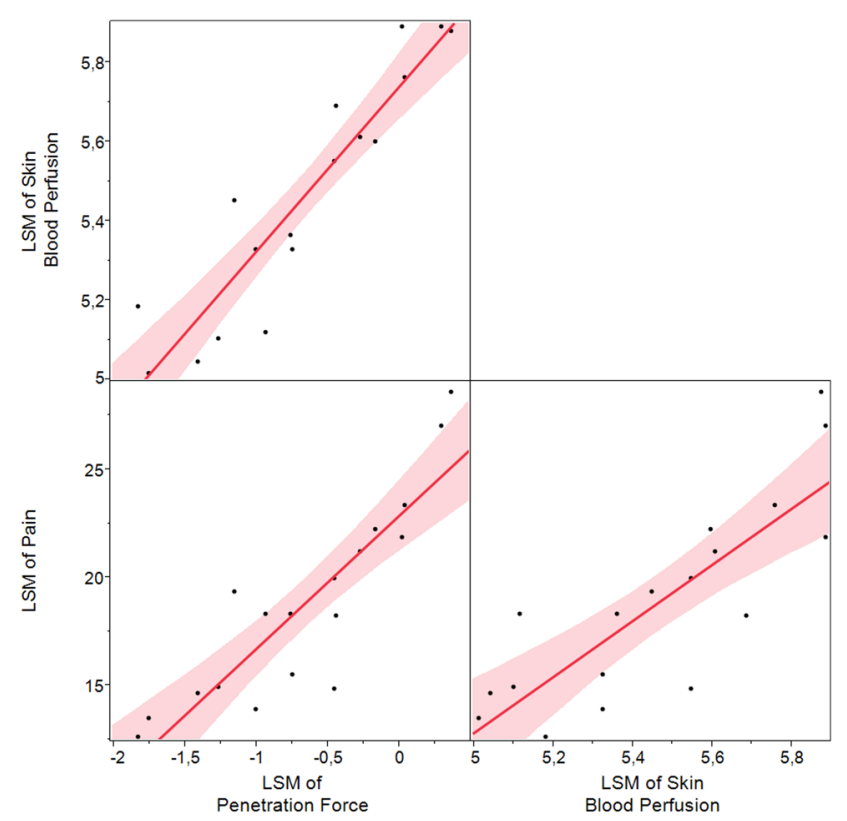

Figure 2 LSM scatter plot matrix with linear fits of relations between PF, pain, and SBP. PF and SBP values are log-transformed. LSM, least square mean; PF, penetration force; SBP, skin blood perfusion.

\section{Experimental design}

Overall, the two influencing factors on PF, SBP and pain were the subject variation $(p<0.0001$ for all assessments $)$ and the type of needle $(\mathrm{p}<0.0001, \mathrm{p}<0.0001$, and $\mathrm{p}=0.0003$, respectively). Furthermore, pain perception was not moved over the course of the experiment $(\mathrm{p}=0.1596)$ or influenced by the area on the abdomen $(\mathrm{p}=0.6603)$, subject gender $(\mathrm{p}=0.7773)$, subject age $(\mathrm{p}=0.2110)$, subject BMI $(\mathrm{p}=0.4962)$, subject's usual needle diameter $(\mathrm{p}=0.6382)$, or years with injection treatment $(\mathrm{p}=0.4046)$. The only demographical relation to any of the assessments was that subject BMI had a negative relation to SBP ( $p=0.039$ ) - thus, the larger the $\mathrm{BMI}$, the less increase in SBP after a needle insertion.

\section{DISCUSSION}

Needles for subcutaneous injections are developed to be as safe, pain-free and as comfortable as possible to the users. Despite the large market for subcutaneous needles, the methods for testing needles have not changed for many years.

This study found that the design of $32 \mathrm{G}$ and $34 \mathrm{G}$ needles influences user experience, in terms of ease of insertion (PF), skin trauma (SBP increase) and pain. Furthermore, the findings show that there is a relation between the outcomes of the different measured effects.

\section{Effects of needle design \\ Needle diameter}

The three standard three-grind (NF) needles had positive relations between needle diameter and all the measured effects, and all the 34G versions of the alternate grinded needles scored either numerically or significantly lower on all effect parameters than their $32 \mathrm{G}$ equivalents. This indicates that no matter how the needle tip design is, diameter is an important parameter of the needle with respect to user experience. Our pain findings are in line with previous studies showing that pain decreases with decreasing needle diameter. ${ }^{23} 24$ Nevertheless, in the present study pain score and increase in SBP did not differ significantly between NF-32 and NF-30 needles. In our previous pig study, the magnitude of the SBP increase also related positively to 
needle diameter with no significant difference between NF-32 and NF-30 needles. ${ }^{14}$ Thus, an agreement was seen between pain and SBP data in humans and between SBP data in humans and pigs, which implies a relation.

\section{Needle tip design}

The five-grind (BD-32) and asymmetrical three-grind (TER-34) needles caused smaller peak PF in skin and PUR than the NF-32 needle. This is in line with a previous study showing a reduced PF in PUR by the BD-32 needles. ${ }^{5}$ Important to note for these needles are, however, that not only did they vary in tip design, but the NF-32 and TER-34 needles are tapered needles, and the TER-34 is $34 \mathrm{G}$ at the tip, so more than one design factor could potentially influence the difference in $\mathrm{PF}$. However, since the peak PF occurs when the needle tip penetrates the skin, ${ }^{11}{ }^{13}$ the base diameter of the needles should not influence the peak PF. Therefore, the differences seen for PF must be due to the different grinds and/or the tip diameter. Since a smaller PF would have been expected from a $34 \mathrm{G}$ tip needle than from a $32 \mathrm{G}$ needle, the asymmetrical grind on the TER-34 needle may in itself cause larger PF than the five-grind on the BD-32 needle.

The short-tip three-grind (HN3) needles included in this study were probably too obtuse and created PF and SBP increases which were larger than the regular threegrind (HN-32) needle.

The one-grind short-tip (HN1SH) and one-grind long-tip (HN1LO) needles do not have a sharp tip, but a rounded sharp edge. The TER-34 needle has an asymmetrical tip having a partly rounded 'cutting' edge, ${ }^{19}$ so further investigations of a one-grind needle should be made. Since there is no sharp tip, the PF was expected to be higher, which was also the case for all the onegrind needles. However, the one-grind long-tip needle in $34 \mathrm{G}$ (HN1LO-34) did not differ from the HN-32 needle in either SBP or pain. This indicates that the length of the needle grind is a decisive parameter in terms of user experience.

The two-grind needles (HN2) were manufactured to place a side-grind on the one-grind needle as an attempt of making a 'sharp tip' on the needle. The angles we chose, however, actually only turned the needle into a poorer alternative than the one-grind needle in terms of all effect parameters. To make twogrind needles perform on par with standard three-grind needles, the grind angles should be changed.

In general, when excluding the HN2-32 needle, none of the alternative tip designs caused significant differences in pain intensity from the standard three-grind needle. This might be due to inability of the subjects to distinguish between the different needles when needles are smaller than a certain size. For the alternative tip designs, the largest difference in pain which did not meet statistical significance was $6.77 \mathrm{~mm}$. It has been argued that only differences of $10 \mathrm{~mm}$ or more on the
$100 \mathrm{~mm}$ VAS are likely to reflect a true change in the experience of pain. ${ }^{25}{ }^{26}$ Thus, our results indicate that for $32 \mathrm{G}$ and $34 \mathrm{G}$ needles, the blinded perception of pain intensity does not differ between needle designs.

\section{Worn-off silicone}

When needle reuse was simulated by worn-off silicone (NOSILIC), the peak PF was higher than on a fully lubricated needle since friction in the skin is increased. Also the increase in SBP was higher for the NOSILIC needle, which might be due to increased friction creating a 'pull' in the skin. Thereby a larger mechanical stress is exerted on the tissue, leading to a higher degree of degranulation of mast cells and release of, for example, histamine and nitric oxide. ${ }^{27}$ Since both of these compounds are known vasodilators, ${ }^{27} 28$ this might be the cause of the higher increase in SBP. The lack of silicone also did not influence pain, indicating that the sensory nerve endings are not affected by the increased friction in skin. This is in line with a published study concluding that needle reuse is not associated with increased pain, ${ }^{29}$ but contrary to publications claiming that lack of cannula lubrication influences perceptions of pain. ${ }^{50}$

\section{Hooks}

The hooked needles caused higher peak PF than an undamaged needle, probably due to the missing sharp tip and a larger surface area puncturing the skin. The increase in SBP was also higher for both hooked needles, but only the large hooked needles (HOOK150) caused higher pain than a new needle. Thus, minor needle wear, such as worn-off silicone and minor tip damages, may cause the user to feel increased friction when inserting and withdrawing the needle, but this will not necessarily cause more pain.

\section{Effect relations}

This study confirmed that pain is very subjectdependent, but when the intersubject variance was accounted for, the difference in pain perception was better predicted by differences in SBP increase around needle insertion sites than the differences in peak $\mathrm{PF}$ in skin. This means that the SBP increase, which is seen after a needle insertion, is more likely to correctly predict needle pain perception than the peak PF in skin. The reason for the relation between pain and increase in SBP might be that SBP increase is related to the amount of tissue trauma that a needle causes, and that tissue trauma is related to pain. Alternatively, the signal generation of pain triggers the release of vasodilators locally in the tissue, whereby blood perfusion is increased in the area.

When comparing the SBP measurements of the present study to those previously obtained in pigs, ${ }^{14}$ the peak SBP increases and the AUC differences between different diameter needles are very similar in magnitude and in relation to different needles. Thus, SBP 
measurements on pigs can be used to predict SBP in humans, enabling future test of needle performance outside of clinical trials.

Since PF in skin is related to pain perception, and since PF in skin and PUR are correlated, it seems reasonable to assume that PF in PUR is related to pain perception. PUR PF has very little variance and is thereby more sensitive to differences in needle design than $\mathrm{PF}$ in skin, which has higher variance. Likewise, $\mathrm{PF}$ in skin is more sensitive to needle design differences than pain is, as seen for the comparison of the NF-32, BD-32 and TER-34 needles. Thus, pain cannot be predicted by PF in PUR alone. However, if PF in PUR is combined with SBP tests on, for example, pigs, a better prediction of pain perceptions might result. Furthermore, PF reveals ease of penetration through skin, which likely adds to the needle experience as a whole when people with diabetes are inserting the needles themselves.

\section{Strengths and limitations}

Blinding of test subjects was chosen as an attempt to make the pain ratings as objective as possible and unaffected by other circumstances than the actual pain sensation. Thus, subjects could not see which needle was used for insertions, and could not see the action of insertion. On a daily treatment basis, several factors are likely to add to the 'pain' sensation altogether, including ease of insertion, perceived pain, and possible bleeding or skin trauma after the insertion. Thus, future clinical trials of needle testing could, apart from the blinded testing, include a part where the subjects would perform insertions of the needles themselves. Here a more nuanced, but highly relevant, perception of 'pain' would probably emerge.

As expected there was a large intersubject variance in pain, but this was also the case for SBP and PF data.

All needles were tested twice on all subjects, and PF and pain perceptions were measured for all insertions, which provide information of intrasubject variance. The intrasubject variance is used by the statistical model to give a better prediction of the data estimates.

To decrease potential variance effects, we chose to use injection-treated subjects with type 2 diabetes. Subjects naive to needle insertions could have added a factor of needle fear, which potentially could influence both pain perception and SBP, since nervousness can affect blood flow. $^{31} 32$

\section{CONCLUSION}

Needle design in terms of needle diameter and obtuse tip designs were positively related to skin $\mathrm{PF}$, pain, and skin trauma. Thus, smaller needle diameter and more robust alternative tip designs should be used in combination when developing new needles in order to provide better user experiences. Minor side effects of reuse (worn-off silicone and small hooks) may not necessarily affect the user experience, whereas large hooks increase
PF, skin trauma and pain. PFs in skin and in PUR are linearly related. Increased skin trauma and pain perception after needle insertions positively relate to each other, enabling future performance testing of needles outside of a clinical setting.

Acknowledgements The authors thank Søren Andersen, Novo Nordisk A/S, for helping with the statistics, Desirée Sørensen, laboratory technician at the Department of Biomedical Sciences, University of Copenhagen for all your help with acquiring laboratory equipment. The authors also thank all of the employees, PhD students and post doc students at the Department of Biomedical Sciences for sharing their knowledge about clinical trials.

Contributors KAP planned and executed the experiment, carried out data analysis and statistics, and wrote the manuscript. MLJ and NBM helped planning the experiment, contributed to the discussion, and reviewed/edited the manuscript. JK helped with statistics and reviewed/edited the manuscript. BMS helped planning and executing the experiment, contributed to the discussion, and reviewed/edited the manuscript. KAP is the guarantor of this manuscript.

Funding This work was supported by the Innovation Fund Denmark as well as Novo Nordisk A/S as part of the Danish Industrial PhD programme.

Competing interests KAP, MLJ, NBM, and JK are all employees of Novo Nordisk A/S and hold stock in Novo Nordisk A/S.

Ethics approval The study received ethical approval by the Regional Committee of Danish Health Research Ethics (H-6-2014-042) and health authority approval by the Danish Health and Medicines Authority (journal number 2014071250, EUDAMED CIV-ID no. CIV-14-07-012361).

Provenance and peer review Not commissioned; externally peer reviewed.

Data sharing statement No additional data are available.

Open Access This is an Open Access article distributed in accordance with the Creative Commons Attribution Non Commercial (CC BY-NC 4.0) license, which permits others to distribute, remix, adapt, build upon this work noncommercially, and license their derivative works on different terms, provided the original work is properly cited and the use is non-commercial. See: http:// creativecommons.org/licenses/by-nc/4.0/

\section{REFERENCES}

1. Diabetes Atlas [article online], 2015. http://www.idf.org/diabetesatlas (accessed 11-04 2015).

2. Hart JT. Rule of halves: implications of increasing diagnosis and reducing dropout for future workload and prescribing costs in primary care. Br J Gen Pract 1992;42:116-19.

3. Smith WC, Lee AJ, Crombie IK, et al. Control of blood pressure in Scotland: the rule of halves. BMJ 1990;300:981-3.

4. Weinger K, Beverly EA. Barriers to achieving glycemic targets: who omits insulin and why? Diabetes Care 2010;33:450-2.

5. Hirsch L, Gibney M, Berube J, et al. Impact of a modified needle tip geometry on penetration force as well as acceptability, preference, and perceived pain in subjects with diabetes. J Diabetes Sci Technol 2012;6:328-35.

6. Hirsch LJ, Gibney MA, Li L, et al. Glycemic control, reported pain and leakage with a $4 \mathrm{~mm} \times 32 \mathrm{G}$ pen needle in obese and non-obese adults with diabetes: a post hoc analysis. Curr Med Res Opin 2012;28:1305-11.

7. Strock E, Bergenstal R, Parvu V, et al. Glycemic control, pain and leakage with $4 \mathrm{~mm}$ vs larger pen needles in obese patients treated

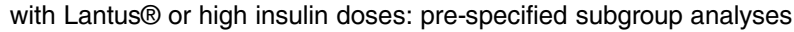
Diabetes 2013;62:A255.

8. Heise T, Nosek L, Dellweg S, et al. Impact of injection speed and volume on perceived pain during subcutaneous injections into the abdomen and thigh: a single-centre, randomized controlled trial. Diabetes Obes Metab 2014;16:971-6.

9. Asakura $\mathrm{T}$, Seino $\mathrm{H}$, Nunoi K, et al. Usability of a microtapered needle (TN3305) for insulin treatment in Japanese patients with diabetes mellitus: a comparative clinical study with a standard thin wall needle. Diabetes Technol Ther 2006;8:489-94.

10. Iwanaga M, Kamoi K. Patient perceptions of injection pain and anxiety: a comparison of NovoFine 32 -gauge tip $6 \mathrm{~mm}$ and Micro 
Fine Plus 31-gauge $5 \mathrm{~mm}$ needles. Diabetes Technol Ther 2009;11:81-6.

11. Kinast P. Quality testing of medical needles-laboratory and practice. Med Device Technol 1992;3:46-9.

12. Kinast $\mathrm{P}$, Andreasson $\mathrm{K}$, Jorgensen $\mathrm{E}$, et al. Needle for penetrating a membrane. U.S. Patent No. 8,328,772. 11 Dec 2012.

13. Mayer G, Knappertz V, Kinast P. Needles: a comparison study. Med Device Technol 2009;20:30, 32-34.

14. Præstmark KA, Jensen CB, Stallknecht B, et al. Skin blood perfusion and cellular response to insertion of insulin pen needles with different diameters. J Diabetes Sci Technol 2014;8:752-9.

15. Boas DA, Dunn AK. Laser speckle contrast imaging in biomedical optics. J Biomed Opt 2010;15:011109.

16. Briers JD. Laser Doppler, speckle and related techniques for blood perfusion mapping and imaging. Physiol Meas 2001;22:R35-66.

17. Draijer M, Hondebrink E, van Leeuwen $\mathrm{T}$, et al. Review of laser speckle contrast techniques for visualizing tissue perfusion. Lasers Med Sci 2009;24:639-51.

18. Murray AK, Moore TL, Manning JB, et al. Noninvasive imaging techniques in the assessment of scleroderma spectrum disorders. Arthritis Rheum 2009;61:1103-11.

19. Nanopass $₫ 34 G$ [article online], 2015. http://www.terumo-europe. com/en-emea/diabetes-management/pen-needles/nanopass $\% C 2 \%$ AE-34g (accessed 11-04 2015).

20. Strauss K, Gols HD, Hannet I, et al. A pan-European epidemiologic study of insulin injection technique in patients with diabetes. Pract Diab Int 2002;19:71-6.

21. Vardar B, Kizilci S. Incidence of lipohypertrophy in diabetic patients and a study of influencing factors. Diabetes Res Clin Pract 2007;77:231-6.
22. Mahé G, Humeau-Heurtier A, Durand S, et al. Assessment of skin microvascular function and dysfunction with laser speckle contrast imaging. Circ Cardiovasc Imaging 2012;5:155-63.

23. Arendt-Nielsen L, Egekvist $\mathrm{H}$, Bjerring P. Pain following controlled cutaneous insertion of needles with different diameters. Somatosens Mot Res 2006;23:37-43.

24. Egekvist $\mathrm{H}$, Bjerring $\mathrm{P}$, Arendt-Nielsen L. Pain and mechanical injury of human skin following needle insertions. Eur J Pain 1999;3:41-9.

25. Powell CV, Kelly AM, Williams A. Determining the minimum clinically significant difference in visual analog pain score for children. Ann Emerg Med 2001;37:28-31.

26. Bijur PE, Silver W, Gallagher EJ. Reliability of the visual analog scale for measurement of acute pain. Acad Emerg Med 2001;8:1153-7.

27. Rang HP, Dale MM, Ritter JM, et al. Rang and Dale's pharmacology. Edinburgh: Churchill Livingstone, 2012.

28. Palmer RM, Ferrige AG, Moncada S. Nitric oxide release accounts for the biological activity of endothelium-derived relaxing factor. Nature 1987;327:524-6.

29. De Coninck C, Frid A, Gaspar R, et al. Results and analysis of the 2008-2009 Insulin Injection Technique Questionnaire survey. $J$ Diabetes 2010;2:168-79.

30. Hirsch LJ, Gibney MA, Albanese J, et al. Comparative glycemic control, safety and patient ratings for a new $4 \mathrm{~mm} \times 32 \mathrm{G}$ insulin pen needle in adults with diabetes. Curr Med Res Opin 2010;26:1531-41.

31. Söderström T, Stefanovska A, Veber M, et al. Involvement of sympathetic nerve activity in skin blood flow oscillations in humans. Am J Physiol Heart Circ Physiol 2003;284:H1638-1646.

32. Wallin BG. Neural control of human skin blood flow. J Auton Nerv Syst 1990;30(Suppl):S185-190. 